\title{
Pulmonary Tuberculosis in Older Adults: Increased Mortality Related to Tuberculosis Within Two Months of Treatment Initiation
}

\author{
Isano Hase ${ }^{1,2}$ (1) $\cdot$ Katelynne Gardner Toren ${ }^{3} \cdot$ Hitomi Hirano $^{1} \cdot$ Kimiko Sakurai $^{4}$. David J. Horne ${ }^{3,5} \cdot$ Takefumi Saito $^{1}$. \\ Masahiro Narita ${ }^{3,5}$
}

Accepted: 13 June 2021 / Published online: 5 July 2021

(c) The Author(s), under exclusive licence to Springer Nature Switzerland AG 2021

\begin{abstract}
Background The proportion of tuberculosis (TB) patients who are older adults is increasing worldwide. Nearly $60 \%$ of TB patients in Japan are 70 years or older, and the TB incidence rate in Japan is one of the highest among high-income countries. The previous TB treatment guidelines prior to 2018 in Japan recommended excluding pyrazinamide (PZA) from the initial regimen for patients aged over 80 years.

Objectives We aimed to examine differences in TB treatment outcomes among different age groups, and between those who received PZA and those who did not.

Methods We performed a retrospective cohort study of patients with pulmonary TB who were managed at a single medical center in Japan. We compared treatment outcomes and adverse events that resulted in treatment interruption across the age groups.

Results Of 246 patients, 117 (48\%) were aged 75 years or older. Compared with patients aged $<74$ years, those $\geq 75$ years were less likely to have PZA in the initial regimen $(53.0 \%$ vs $89.9 \% ; p<0.0001)$, more likely to die during treatment $(38.5 \%$ vs $6.2 \% ; p<0.0001)$, and more likely to experience adverse events $(30.8 \%$ vs $19.4 \% ; p<0.05)$. The mortality rate related to TB at 2 months after TB treatment initiation was $28 \%$ in those aged $\geq 84$ years. Furthermore, among patients aged $\geq 84$ years, those who did not receive PZA were significantly more likely to die than those who did $(65.8 \%$ vs $36.8 \% ; p<0.05)$. Conclusions Patients aged $\geq 75$ years with pulmonary TB experienced increased mortality related to TB during treatment and more frequent adverse events than younger patients, even though PZA was often avoided among older patients.
\end{abstract}

Isano Hase

isanotaki@gmail.com

1 Department of Respiratory Medicine, National Hospital Organization Ibarakihigashi National Hospital, 825, Terunuma. Tokai-mura, Naka-gun, Ibaraki 319-1113, Japan

2 Department of Pulmonary Medicine, Saitama City Hospital, Saitama, Japan

3 TB Control Program, Public Health-Seattle \& King county, Seattle, WA, USA

4 Department of Health Information Management, National Hospital Organization Ibarakihigashi National Hospital, Ibaraki, Japan

5 Division of Pulmonary, Critical Care and Sleep Medicine, University of Washington, Seattle, WA, USA

\section{Key Points}

As the global population ages, the proportion of tuberculosis (TB) patients who are older adults is also increasing around the world, especially in high-income countries.

Aging is one of the risk factors that are associated with poor outcomes of pulmonary TB.

Older patients with pulmonary TB have high mortality; death related to TB was observed in $38 \%$ of those aged $\geq 75$ years during the course of treatment and in particular, $28 \%$ of those aged $\geq 84$ years within 60 days of treatment initiation.

Older TB patients experienced adverse events more frequently despite the common practice of excluding pyrazinamide from the initial regimen among older patients in Japan. 


\section{Introduction}

As the global population ages [1], the proportion of tuberculosis (TB) that occurs in elder patients is increasing, especially in high-income countries $[2,3]$. Japan has one of the largest older populations in the world [4], with $28.1 \%$ of the population aged 65 years and older in 2018 [5]. In the same year, 15,590 new TB cases were reported in Japan (12.3 cases per 100,000) [6]. This was the lowest TB incidence rate since 1999, but it remains higher than most high-income countries. Among these TB patients, 59.9\% were age 70 years or older, while patients aged $80-89$ years were the most frequently represented decile $(29.1 \%)$ among ten age categories from the first to the tenth decade of life.

There are two unique aspects of TB management in Japan. First, TB patients with acid-fast bacilli smear-positive sputum are required by law to be hospitalized until they are no longer infectious based on smear status. In addition, regardless of smear status, it is common practice to hospitalize older patients with pulmonary TB at the beginning of TB treatment. Hospitalization of patients for the initiation of TB treatment enables close monitoring for adverse events due to TB medications and supports intensive management of comorbidities such as diabetes mellitus. The median duration of TB patients' hospitalization is approximately 2 months [6]. Second, until 2018, the Japanese TB treatment guidelines recommended excluding pyrazinamide (PZA) from TB regimens for patients older than 80 years of age [7]. Cautious recommendations for the use of PZA in patients aged over 75 years are in the US guideline [8] and the exclusion of PZA among older patients is common worldwide [9-11]. Exclusion of PZA among patients aged $80-84$ years and $>90$ years in 2009-2014 in Japan was > 75\% and 90\%, respectively.

We performed a retrospective cohort study of patients treated for pulmonary TB at a single Japanese hospital in order to examine differences in TB treatment outcomes by age. An additional goal was to evaluate the exclusion of PZA on treatment outcomes. We hypothesized that patients 75 years of age and older would have greater mortality during TB treatment compared with patients younger than 75 years. Preliminary results of this study have been previously reported in the form of an abstract [12].

\section{Methods}

\subsection{Study Design}

We examined treatment outcomes of adults diagnosed with pulmonary TB who initiated TB treatment between January 1, 2009 and December 31, 2014 at National Hospital
Organization Ibarakihigashi National Hospital in Ibaraki Prefecture, Japan. This hospital provides both inpatient and outpatient-based care of patients with TB. We excluded patients who were lost to follow-up, were under the age of 18 years, or were diagnosed with multidrug-resistant TB (MDR-TB; resistant to at least isoniazid and rifampin). Medical records were reviewed and the following data were abstracted: age at TB diagnosis, birth sex, race/ethnicity, country of birth (Japan vs outside Japan), comorbidities (diabetes, end-stage renal disease, human immunodeficiency virus [HIV], other immunosuppression), microbiological results, cavitation on chest radiograph, site of TB disease, information on TB treatment including interruptions, treatment outcome, and, if applicable, cause of death (death related to TB vs unrelated to TB). 'Death related to TB' includes death related to TB disease and/or TB treatment. Since approximately $50 \%$ of our cohort included patients aged over 75 years, it was often difficult to differentiate whether symptoms or laboratory abnormalities were due to adverse events related to TB treatment or other causes.

During the first 2 months of treatment, blood chemistry, complete blood count, and sputum for acid-fast bacilli smear and culture were obtained at least every 2 weeks, and a chest radiograph was obtained at least once a month.

We used the definition set forth by the Report of Verified Case of Tuberculosis of the US Centers for Disease Control and Prevention [13] to differentiate 'death related to TB' versus 'death unrelated to TB'. Significant adverse events for this study were ascertained by searching for interruptions in one or more drugs during TB treatment. For those who had adverse events, the following determinations were made: the onset of the adverse event, the impact of the adverse event (refer to the international WHO definitions of adverse drug reactions [14]: 'modest'-medications permanently discontinued and/or adverse effect requiring additional medication; or 'severe' - requiring hospitalization from outpatient-based care, or death), type of the adverse event (hypersensitivity, liver injury, gastrointestinal intolerance, musculoskeletal, or other), medications responsible for the adverse event, and level of certainty that the medication was responsible ('confirmed' based on drug challenges, or 'high level of certainty' based on resolution of the adverse event after medication changes while other causes were unlikely). Where there was polytherapy, the responsible drug was determined based on the pattern of adverse events (e.g., liver injury; hepatocellular injury vs cholestatic pattern) or based on re-challenge of drugs. 'Multiple adverse events' was defined as two or more adverse events resulting in treatment interruption at different times during treatment. The assessment documented by the primary physician managing the patient was used; if it was not clearly documented, the study investigators $(\mathrm{IH}, \mathrm{HH}$, TS) made a decision. 
Patients were categorized into the following age groups: $18-44,45-64,65-74,75-83$, and $\geq 84$ years. The median age among the older patients over 75 years was 84 years. Patients whose initial regimen consisted of isoniazid, rifampin, PZA and ethambutol were considered to have been started on the standard TB regimen ('HRZE'). The physicians who primarily managed the patients decided whether to include PZA in the initial regimen. 'Time to sputum culture conversion' was calculated using the date when a specimen was collected for the first consistently negative sputum culture results minus the date TB treatment started. 'Time to treatment completion' was calculated only for those who successfully completed treatment and was grouped into $<26$ weeks, 26 to $<39$ weeks, 39 to $<52$ weeks, and 52 weeks or more. Treatment completion was verified by the TB clinician who cared for the patient. For those who died during TB treatment, 'time to death' was calculated using the date of death minus the date TB treatment was started.

\subsection{Statistical Analyses}

Statistical analyses were conducted using SAS 9.3. We used chi-square tests, Fisher's exact test, or ANOVA to determine differences in patient characteristics and outcomes by patient age. Our primary analysis compared patients aged $\geq 75$ years with those $<75$ years. Secondary analyses evaluated TB treatment outcomes of the oldest patients ( $\geq 84$ years) compared with younger patients. We constructed Kaplan-Meier survival plots of time from TB treatment initiation to last adverse event and time to death (deaths related to TB as the primary analysis, and all-cause mortality as the secondary analysis) stratified by age groups. Lines were truncated at time after which no further adverse events occurred. Cox proportional hazard regression and log-rank test were used to estimate the hazard ratios of all-cause mortality, deaths due to TB or TB treatment, and adverse events related to TB medications. All $p$-values were two-tailed.

\section{Results}

\subsection{Patient Characteristics}

A total of 272 TB patients aged 18 years or older were started on TB treatment at Ibarakihigashi National Hospital from January 2009 through December 2014. We excluded two patients with MDR-TB and 24 patients with extra-pulmonary TB, resulting in 246 patients eligible for our analysis (Fig. 1). Seven patients were started on TB treatment as outpatients and 239 started TB treatment while hospitalized. Among all patients, 117 (48\%) were aged 75 years or older, $35.4 \%$ were women, all identified as Asian, and most (96.3\%) were born in Japan. There was no significant difference in the prevalence of co-morbidities that are risk factors for TB progression in patients aged $\geq 75$ years compared with those $<75$ years $(18.8 \%$ vs $16.3 \% ; p=0.62)$ (Table 1$)$.

Patients aged $\geq 75$ years were significantly more likely than those $<75$ years to be culture positive $(93.2 \%$ vs $81.4 \%$; $p<0.01$ ), but there was no significant difference between those aged $\geq 75$ years and $<75$ years in sputum smear positivity ( $56.4 \%$ vs $48.8 \% ; p=0.23$ ), cavitation on chest radiographs $(27.4 \%$ vs $34.9 \% ; p=0.20)$, or the proportion of those with delayed culture conversion beyond 2 months of TB treatment (17.8\% vs $19.6 \% ; p=0.75)$ (Table 2). Patients aged $\geq 75$ years were more likely to have PZA withheld (47.0\% vs $10.1 \% ; p<0.0001)$. Compared with patients $<75$ years, those $\geq 75$ years were less likely to complete TB treatment (61.5\% vs $91.5 \% ; p<0.0001)$, and only $43.9 \%$ of patients $\geq 84$ years completed treatment. Although the mean time to treatment completion was significantly longer for patients aged $\geq 75$ years compared with those $<75$ years (274 days vs 246 days; $p \leq 0.05$ ), there was no difference in time to treatment completion when restricting to patients who had PZA in their initial regimen (247 days vs 242 days; $p=0.68)$.
Fig. 1 Flow chart of study population. $T B$ tuberculosis
Pulmonary TB patients who initiated TB treatment at Ibarakihigashi National Hospital between Jan 2009 - Nov $2014 \quad(n=272)$

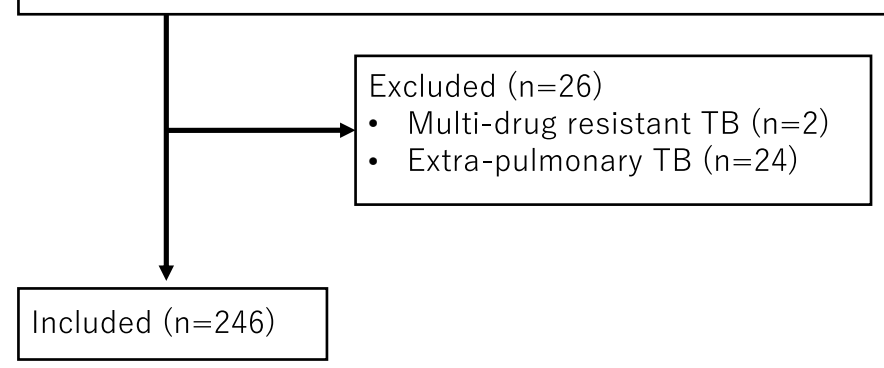




\subsection{Mortality}

Patients aged $\geq 75$ years were more likely to die during treatment than those $<75$ years $(38.5 \%$ vs $6.2 \%$; $p<0.0001)$. This difference was greater for patients $\geq 84$ years compared with those $<84$ years $(56.1 \%$ vs $11.1 \%$; $p<0.0001)$. Among patients aged $\geq 84$ years, those who did not receive PZA were significantly more likely to die than those who did (65.8\% vs $36.8 \% ; p<0.05)$.

Of all deaths, $85 \%$ were related to $\mathrm{TB}$, such as failure to thrive due to TB disease. Only one case died of severe liver injury due to TB treatment, likely because of rifampin. The risk of death related to TB was significantly higher among patients aged 75-83 years (HR 10.09; $p<0.05$ ) and aged 84 or older (HR 28.26; $p<0.01$ ) compared with patients aged 18-44 years (Table 3 ). There were significant differences across age groups in time until death related to TB (Fig. 2 and Table $\mathrm{S} 1$ in the electronic supplementary material [ESM]). In addition, the differences across the age groups were more pronounced when the time to death from any cause was compared ( $\geq 84$ years: HR 34.23 ; 75-83: HR 10.95; 65-74: HR 8.78; 45-64: HR 0.97; $p<0.0001)$. Significant differences in mortality related to TB by age group were observed as early as 60 days following TB treatment initiation (Table 4), including an observed mortality of $28 \%$ among those aged $\geq 84$ years.

\subsection{Adverse Events}

Patients aged $\geq 75$ years compared with those $<75$ years were significantly more likely to have adverse events resulting in treatment interruption due to any cause $(30.8 \%$ vs $19.4 \% ; p<0.05)$; this difference was not significant when restricting to adverse events attributable to TB medications (27.4\% vs $19.4 \%$; $p=0.14$ ) (Table 5 ). The overall rates of adverse events per person-months on treatment were 7.56 adverse events/100 person-months for those aged 84 years and older, 4.70 adverse events/100 person-months for those aged 75-83 years, 4.76 adverse events/100 person-months for those aged 65-74 years, 2.89 adverse events/100 person-months for those aged 45-64 years, and 1.17 adverse events/100 person-months for those aged 18-44 years. When we restricted the analysis to adverse events attributable to TB medications, liver injury was the most common adverse event across all age groups. PZA was significantly more likely to be the cause of a first adverse event in those aged $<75$ years as compared with those $\geq 75$ years $(32.0 \%$ vs $6.3 \% ; p<0.05)$, though fewer patients aged $\geq 75$ years received PZA as part of the initial regimen. The proportion of patients aged $\geq 75$ years who had liver injury was not significantly different between those who started and those who did not start PZA (35.7\% vs $66.7 \% ; p=0.08)$.

The risk of adverse events due to TB medications was significantly higher for those aged 65-74, 75-83, and $\geq 84$ years compared with those 18-44 years (Table 6). There was no significant difference across age groups in time

Table 1 Characteristics of the cross-sectional population by different age groups $(N=246)$

\begin{tabular}{|c|c|c|c|c|c|}
\hline Patient characteristics & $18-44$ y $(n=46)$ & $45-64$ y $(n=48)$ & $65-74$ y $(n=35)$ & $75-83$ y $(n=60)$ & $\geq 84$ y $(n=57)$ \\
\hline \multicolumn{6}{|l|}{ Birth sex } \\
\hline Male $(\%)$ & $28(60.9)$ & $28(58.3)$ & $29(82.9)$ & $40(66.7)$ & $34(59.7)$ \\
\hline Female $(\%)$ & $18(39.1)$ & $20(41.7)$ & $6(17.1)$ & $20(33.3)$ & $23(40.4)$ \\
\hline \multicolumn{6}{|l|}{ Country of birth ${ }^{\mathrm{a}}$} \\
\hline Japan $(\%)$ & $39(84.8)$ & $48(100)$ & $35(100)$ & $58(96.7)$ & $57(100)$ \\
\hline Outside Japan (\%) & $7(15.2)$ & $0(0)$ & $0(0)$ & $2(3.3)$ & $0(0)$ \\
\hline \multicolumn{6}{|l|}{ Race/ethnicity } \\
\hline Asian (\%) & $46(100)$ & $48(100)$ & $35(100)$ & $60(100)$ & $57(100)$ \\
\hline \multicolumn{6}{|l|}{ Co-morbidities (TB risk factors) } \\
\hline Diabetes mellitus (\%) & $4(8.7)$ & $8(16.7)$ & $6(17.1)$ & $9(15.0)$ & $11(19.3)$ \\
\hline End-stage renal disease (\%) & $0(0)$ & $1(2.1)$ & $0(0)$ & $0(0)$ & $1(1.8)$ \\
\hline HIV positive (\%) & $1(2.2)$ & $0(0)$ & $0(0)$ & $0(0)$ & $0(0)$ \\
\hline Immunosuppression (non-HIV related) $(\%)$ & $0(0)$ & $2(4.2)$ & $0(0)$ & $1(1.7)$ & $1(1.8)$ \\
\hline Dementia $^{\mathrm{a}, \mathrm{b}, \mathrm{c}}$ & $0(0)$ & $0(0)$ & $1(2.9)$ & $4(6.7)$ & $11(19.3)$ \\
\hline Contact with infectious TB patient & $3(6.5)$ & $5(10.4)$ & $1(2.9)$ & $1(1.7)$ & $0(0)$ \\
\hline
\end{tabular}

$H I V$ human immunodeficiency virus, $T B$ tuberculosis

${ }^{\text {a }} p$-Value $<0.05$ comparing those aged $<65$ to those $\geq 65$ years

${ }^{\mathrm{b}} p$-Value $<0.05$ comparing those aged $<75$ to those $\geq 75$ years

${ }^{c} p$-Value $<0.05$ comparing those aged $<84$ to those $\geq 84$ years 
Table 2 Characteristics of TB treatment and outcomes of the cross-sectional population by different age groups $(N=246)$

\begin{tabular}{|c|c|c|c|c|c|}
\hline TB characteristics & $18-44$ y $(n=46)$ & $45-64$ y $(n=48)$ & $65-74$ y $(n=35)$ & $75-83$ y $(n=60)$ & $\geq 84$ y $(n=57)$ \\
\hline Multi-site TB (\%) & $10(21.7)$ & $6(12.5)$ & $9(25.7)$ & $17(28.3)$ & $10(17.5)$ \\
\hline Smear positive $(\%)$ & $17(37.0)$ & $25(52.1)$ & $21(60.0)$ & $35(58.3)$ & $31(54.4)$ \\
\hline Culture-positive TB $(\%)^{\mathrm{a}, \mathrm{b}}$ & $39(84.8)$ & $36(75.0)$ & $30(85.6)$ & $56(93.3)$ & $53(93.0)$ \\
\hline Cavitation on chest radiograph (\%) & $16(34.8)$ & $15(31.3)$ & $14(40.0)$ & $17(28.3)$ & $15(26.3)$ \\
\hline Use of PZA ${ }^{a, b, c}$ & $42(91.3)$ & $44(91.7)$ & $30(85.7)$ & $43(71.7)$ & $19(33.3)$ \\
\hline Completed therapy $(\%)^{\mathrm{a}, \mathrm{b}, \mathrm{c}}$ & $45(97.8)$ & $45(93.8)$ & $28(80.0)$ & $47(78.3)$ & $25(43.9)$ \\
\hline $\begin{array}{l}\text { Median duration of initial hospitaliza- } \\
\text { tion (days) })^{\text {b,c }}\end{array}$ & 36.5 & 38 & 44 & 53 & 61 \\
\hline Died during therapy $(\%)^{\mathrm{a}, \mathrm{b}, \mathrm{c}}$ & $1(2.2)$ & $1(2.1)$ & $6(17.1)$ & $13(21.7)$ & $32(56.1)$ \\
\hline Death related to TB $(\%)^{\mathrm{a}, \mathrm{b}, \mathrm{c}}$ & $1(2.2)$ & $1(2.1)$ & $5(14.3)$ & $12(20.0)$ & $26(45.6)$ \\
\hline Median time to death (days) & 82 & 10 & 39 & 40 & 48 \\
\hline \multicolumn{6}{|l|}{ Time to treatment completion } \\
\hline Average days ${ }^{\mathrm{a}, \mathrm{b}, \mathrm{c}}$ & 229 & 252 & 264 & 258 & 302 \\
\hline$<26$ weeks $(\%)$ & $0(0)$ & $3(6.7)$ & $0(0)$ & $1(2.1)$ & $0(0)$ \\
\hline 26 to $<39$ weeks (\%) & $32(71.1)$ & $24(53.3)$ & $14(50.0)$ & $26(55.3)$ & $10(40.0)$ \\
\hline 39 to $<52$ weeks (\%) & $11(24.4)$ & $15(33.3)$ & $12(42.9)$ & $17(36.2)$ & $8(32.0)$ \\
\hline$\geq 52$ weeks $(\%)^{\mathrm{c}}$ & $2(4.4)$ & $3(6.7)$ & $2(7.1)$ & $3(6.4)$ & $7(28.0)$ \\
\hline \multicolumn{6}{|l|}{ Sputum culture conversion } \\
\hline Average days & 45.4 & 39.3 & 38.6 & 43.2 & 41.1 \\
\hline$>2$ months $(\%)$ & $7(18.4)$ & $9(25.0)$ & $4(14.3)$ & $9(18.0)$ & $7(17.5)$ \\
\hline
\end{tabular}

$P Z A$ pyrazinamide, $T B$ tuberculosis

${ }^{\text {a }} p$-Value $<0.05$ comparing those aged $<65$ to those $\geq 65$ years

${ }^{\mathrm{b}} p$-Value $<0.05$ comparing those aged $<75$ to those $\geq 75$ years

${ }^{c} p$-Value $<0.05$ comparing those aged $<84$ to those $\geq 84$ years

Table 3 Hazard ratio of death related to tuberculosis by age group

\begin{tabular}{lllr}
\hline Age group (y) & HR & $95 \%$ CI & $p$-Value \\
\hline $18-44$ & Reference & & \\
$45-64$ & 0.97 & $0.06-15.52$ & 0.98 \\
$65-74$ & 7.30 & $0.85-62.50$ & 0.07 \\
$75-83$ & 10.09 & $1.31-77.58$ & 0.03 \\
$84+$ & 28.26 & $3.83-208.43$ & $<0.01$ \\
\hline
\end{tabular}

$H R$ hazard ratio, $95 \%$ CI 95\% confidence interval

until the last adverse event due to TB medications (Fig. 3 and Table $\mathrm{S} 2$ in the ESM; $p=0.09$ ).

\section{Discussion}

In our study, we found that age $\geq 75$ years was associated with an increased risk of death related to TB and from any cause during treatment and that $85 \%$ of deaths during treatment in all age groups were related to TB. Furthermore, the increased risk of death, both related to TB and from any cause, was even greater for patients aged $\geq 84$ years, among whom $28 \%$ experienced death related to TB within 2 months of TB treatment initiation. Those aged $\geq 75$ years were also at increased risk of adverse events due to TB medications. Older TB patients have been reported to have a higher risk of adverse events $[10,15]$. While a prior study by our group in King County, Washington, USA ('the King County study') found that those aged $\geq 75$ years were likely to have adverse events later in therapy compared with other age groups [11], there were no significant differences in time to last adverse events across the age groups in this cohort. The close observation during hospitalization that is common practice in Japan may have increased early detection and intervention to prevent late-onset adverse events during the subsequent treatment period.

As is consistent with a systematic review [16], this study showed that advanced age and co-morbidities are related to poor prognosis of $\mathrm{TB}$ and that early death during $\mathrm{TB}$ treatment is related to TB. A smaller proportion of patients with co-morbidities in this study may unravel advanced age as an independent risk factor for mortality during TB treatment. For example, the prevalence of diabetes among older TB patients aged $\geq 65$ and $\geq 75$ years are is $17 \%$ for both (26 of 152 and 20 of 117, respectively) in the present study, which is notably lower than that of the patients aged $\geq 65$ years in the King County study (40\%, 44 of 111) [11] 


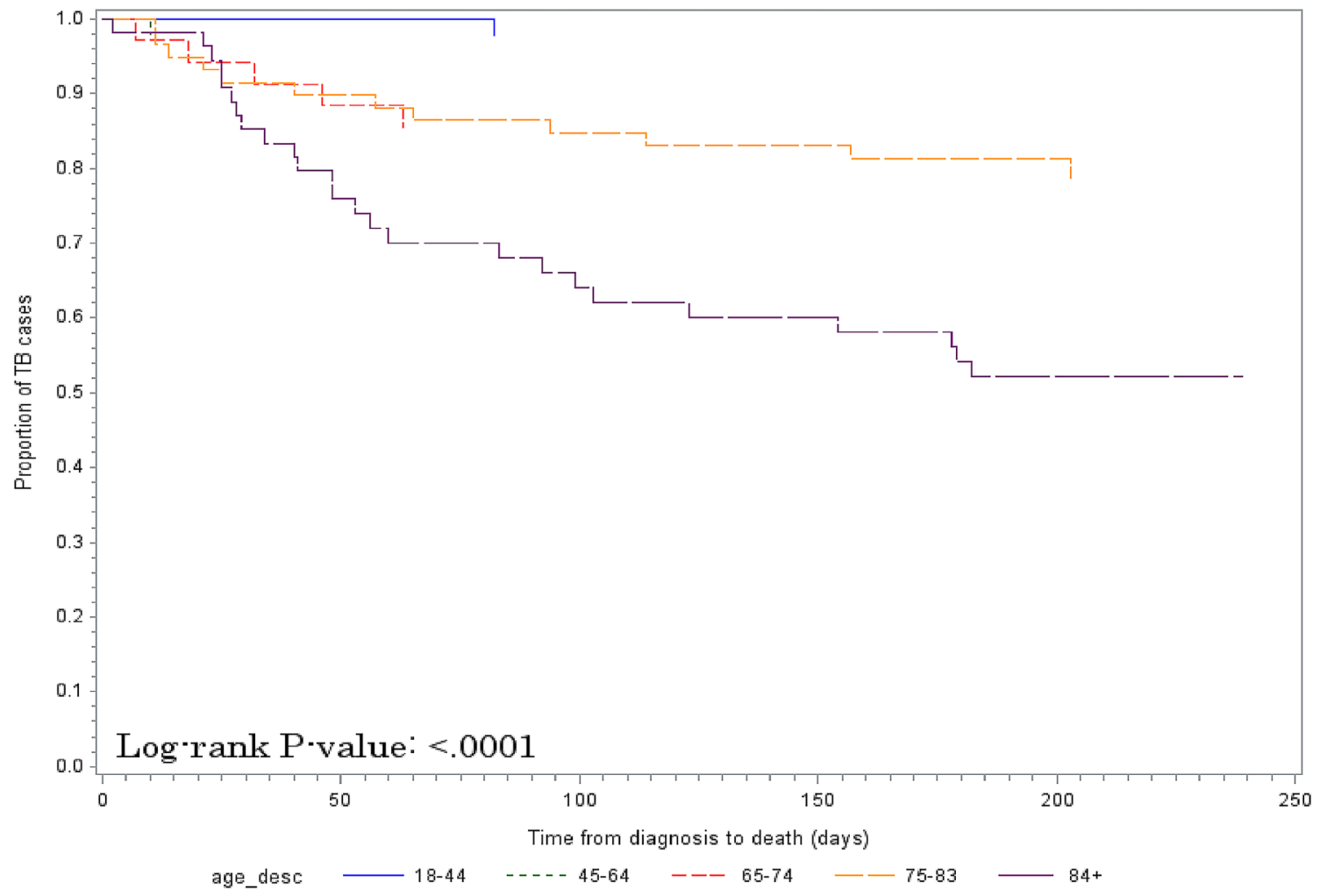

Fig. 2 Differences in time to death related to TB by age group (18-44, 45-64, 65-74, 75-83 and $\geq 84$ years). TB tuberculosis

Table 4 Death related to TB within 60 days of TB treatment initiation by age group

\begin{tabular}{|c|c|c|c|c|c|}
\hline & \multicolumn{5}{|l|}{ Age group (y) } \\
\hline & $18-44$ y $(n=46)$ & $45-64$ y $(n=48)$ & $65-74$ y $(n=35)$ & $75-83$ y $(n=60)$ & $\geq 84$ y $(n=57)$ \\
\hline $\begin{array}{l}\text { The number of patients who died related } \\
\text { to TB within } 60 \text { days of TB treatment } \\
\text { initiation }\end{array}$ & 0 & 1 & 4 & 7 & 16 \\
\hline$\%$ ( $95 \%$ confidence interval) & 0 & $2.1(0.1-11)$ & $11.4(3-27)$ & $11.7(5-23)$ & $28.1(17-42)$ \\
\hline
\end{tabular}

$p<0.0001$

$T B$ tuberculosis

and a report from France showing the prevalence of diabetes in TB patients aged $\geq 75$ years was $29.1 \%$ (32 of 110) [9]. Similarly, the prevalence of co-morbidities such as end-stage renal disease, HIV, and non-HIV-related immunosuppression were also low in the same age group of this cohort. Additionally, the proportions of smear positivity and of presence of cavitation on the chest radiograph had no significant difference in the groups aged $>65$ years compared with the younger ones (Table 2). These characteristics of our cohort cement the notion that advanced age itself is a significant risk factor for TB mortality.

Japan is one of the countries with the highest proportion of those aged over 65 years [4] and $60 \%$ of all TB cases are aged $\geq 70$ years. This high proportion of TB in older patients poses a substantial challenge for TB control in Japan, particularly because genotyping analyses have shown that it mainly results from 'endogenous reactivation' of remote infection, as observed in other high-income countries [17-19]. The TB endemic in Japan peaked in 1940-1950, around World War II, when the current older population were children $[6,18]$. Treatment of latent tuberculosis infection (LTBI) is not generally offered to older patients as the risk of LTBI treatment is perceived to outweigh its benefits. In addition, even though older TB patients rarely lead to clusters, TB transmission to younger persons has been observed in hospitals and nursing homes [19], and atypical presentations of TB among older patients may result in delayed TB diagnosis [3], especially given the current 
Table 5 Characteristics of adverse reactions related to TB medication by different age groups $(N=246)$

\begin{tabular}{|c|c|c|c|c|c|}
\hline & $18-44$ y & $45-64$ y & $65-74$ y & $75-83$ y & $\geq 84 y$ \\
\hline $\begin{array}{l}\text { Patients who experienced any adverse } \\
\text { reactions } \mathrm{s}^{\mathrm{a}, \mathrm{b}}\end{array}$ & 4 & 11 & 10 & 18 & 18 \\
\hline $\begin{array}{l}\text { Patients with adverse reactions due to } \\
\text { TB medications }{ }^{\mathrm{a}}\end{array}$ & 4 & 11 & 10 & 17 & 15 \\
\hline Multiple adverse reactions $\mathrm{s}^{\mathrm{a}, \mathrm{c}}$ & 0 & 0 & $2(20.0)$ & $2(11.8)$ & $5(33.3)$ \\
\hline \multicolumn{6}{|l|}{ Impact of adverse reaction } \\
\hline Modest (\%) & $3(75.0)$ & $6(54.6)$ & $6(60.0)$ & $11(64.7)$ & $11(73.3)$ \\
\hline Severe $(\%)$ & $1(25.0)$ & $5(45.5)$ & $4(40.0)$ & $6(35.3)$ & $4(26.7)$ \\
\hline \multicolumn{6}{|l|}{ Type of first adverse event } \\
\hline GI intolerance $(\%)$ & 0 & 0 & 0 & 0 & 0 \\
\hline Hypersensitivity (\%) & 0 & $5(45.5)$ & $3(30.0)$ & $2(11.8)$ & $2(13.3)$ \\
\hline Liver injury (\%) & $4(100.0)$ & $5(45.5)$ & $5(50.0)$ & $9(52.9)$ & $8(53.3)$ \\
\hline Musculoskeletal (\%) & 0 & 0 & 0 & 0 & 0 \\
\hline Other $^{\mathrm{d}}(\%)$ & 0 & 0 & 0 & $2(11.8)$ & $4(26.7)$ \\
\hline Multiple (\%) & 0 & $1(9.1)$ & $2(20.0)$ & $4(23.5)$ & $1(6.7)$ \\
\hline \multicolumn{6}{|l|}{ Drug responsible for first hold } \\
\hline Ethambutol (\%) & 0 & $1(9.1)$ & $2(20.0)$ & $4(23.5)$ & $3(20.0)$ \\
\hline Isoniazid (\%) & $1(25.0)$ & 0 & $2(20.0)$ & $2(11.8)$ & $1(6.7)$ \\
\hline Pyrazinamide $(\%)^{\mathrm{a}, \mathrm{b}}$ & $2(50.0)$ & $5(45.5)$ & $1(10.0)$ & $1(5.9)$ & $1(6.7)$ \\
\hline Rifampin (\%) & $1(25.0)$ & $1(9.1)$ & $3(30.0)$ & $4(23.5)$ & $5(33.3)$ \\
\hline Unspecified TB drug (\%) & 0 & $4(36.3)$ & $2(20.0)$ & $6(35.3)$ & $3(20.0)$ \\
\hline Other TB drug (\%) & 0 & 0 & $0(0.0)$ & 0 & $2(13.3)$ \\
\hline \multicolumn{6}{|l|}{ Drug level of certainty } \\
\hline Confirmed $(\%)^{\mathrm{a}, \mathrm{b}}$ & 0 & $4(36.4)$ & $1(10.0)$ & 0 & 0 \\
\hline $\operatorname{High}(\%)^{\mathrm{a}, \mathrm{b}}$ & $4(100.0)$ & $7(63.6)$ & $9(90.0)$ & $17(94.4)$ & $15(100)$ \\
\hline
\end{tabular}

GI gastrointestinal, $T B$ tuberculosis

${ }^{\text {a }} p$-Value $<0.05$ comparing those aged $<65$ to those $\geq 65$ years

${ }^{\mathrm{b}} p$-Value $<0.05$ comparing those aged $<75$ to those $\geq 75$ years

${ }^{c} p$-Value $<0.05$ comparing those aged $<84$ to those $\geq 84$ years

${ }^{\mathrm{d}}$ Other includes hypoglycemia, thrombocytopenia, ocular, renal failure, anemia

Table 6 Hazard ratio of adverse events related to TB medication

\begin{tabular}{llll}
\hline Age group (y) & HR & $95 \%$ CI & $p$-Value \\
\hline $18-44$ & Reference & & \\
$45-64$ & 3.00 & $0.95-9.41$ & 0.06 \\
$65-74$ & 3.87 & $1.21-12.34$ & 0.02 \\
$75-83$ & 3.98 & $1.34-11.82$ & 0.01 \\
$84+$ & 3.84 & $1.27-11.60$ & 0.02 \\
\hline
\end{tabular}

95\% CI 95\% confidence interval, $H R$ hazard ratio, $T B$ tuberculosis

COVID-19 pandemic [20, 21]. As our study highlights high mortality related to TB among older patients, the benefit of LTBI treatment in a selected older population can be revisited and the risk-benefit ratio among older LTBI patients should be re-evaluated with the use of safer, short-course rifamycin-based LTBI treatment regimens [22-24]. Further studies on LTBI among older adults is warranted to develop better strategies to reduce mortality and morbidity.

Our study has some limitations. Our finding that among patients aged $\geq 84$ years, withholding PZA was associated with death may be due to confounding by indication, as the primary treating physician decided on the use of PZA on a case-by-case basis. The moderate size of this study may not reach statistical significance. Furthermore, all patients in this study were Asian and the vast majority were born in Japan, potentially limiting generalizability of our findings. Variations in the healthcare system and the standard management of TB patients may influence the clinical outcomes, also impacting generalizability. On the other hand, the homogeneity of the study population might limit confounding factors and thus the results of this study can offer insight into the care and prognosis of older patients with pulmonary TB. 


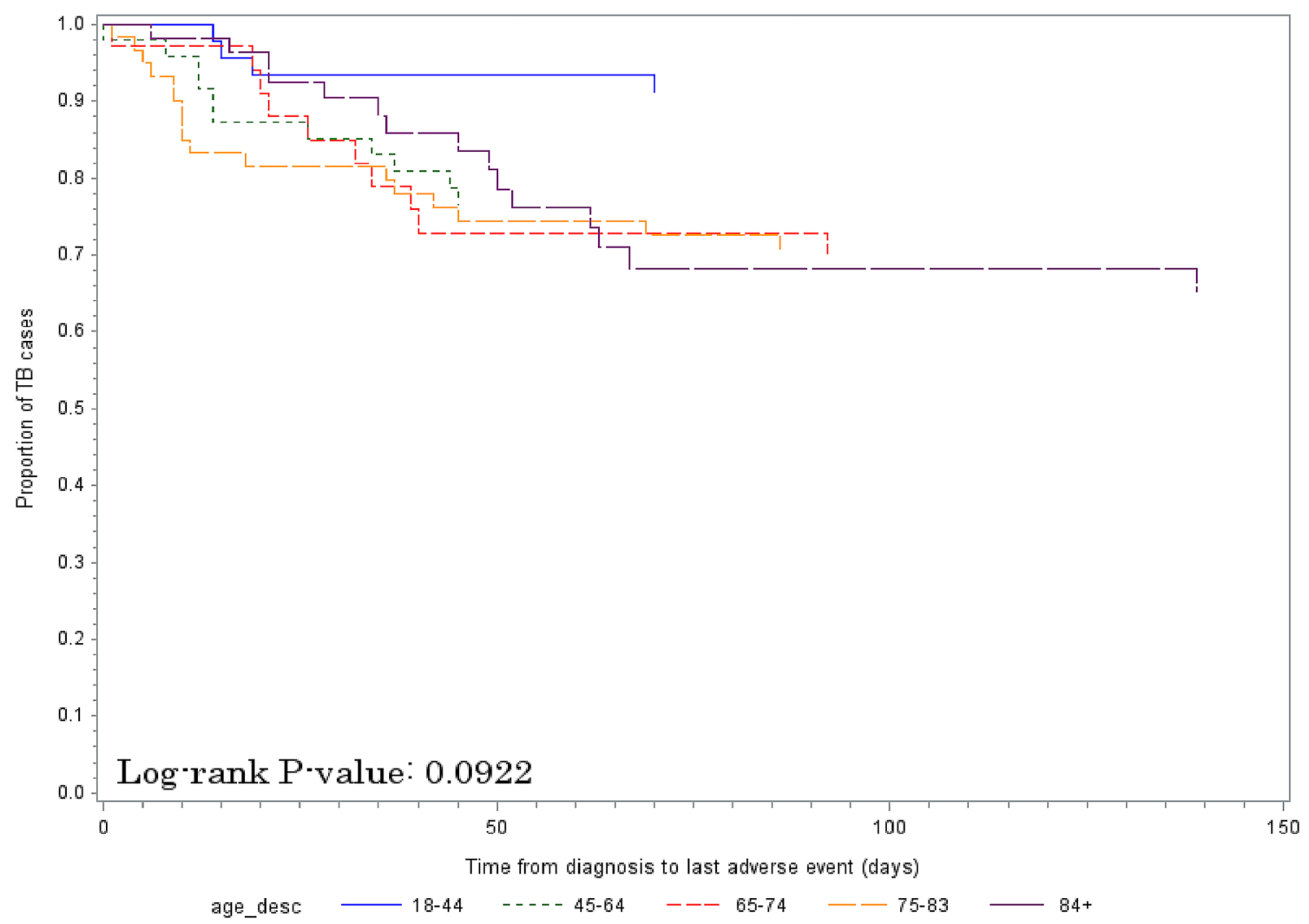

Fig. 3 Differences in time to last adverse event by age group (18-44, 45-64, 65-74, 75-83 and $\geq 84$ years). TB tuberculosis

\section{Conclusions}

The study found increased mortality related to TB among patients aged $\geq 75$ years. Increased mortality in older patients related to TB ( $28 \%$ in those aged $\geq 84$ years) was observed within 60 days of treatment initiation. An increased number of adverse events occurred in older patients despite the common practice of excluding PZA from the initial regimen for many patients aged 80 years and older. As the poor outcomes, including high mortality, ensue when older patients develop TB, the use of safer rifamycin-based regimens for LTBI should be reconsidered in a selected older population.

Supplementary Information The online version contains supplementary material available at https://doi.org/10.1007/s40266-021-00880-4.

Acknowledgements The authors thank Shuji Ohishi, MD, PhD (National Hospital Organization Ibarakihigashi National Hospital) for critical feedback on the manuscript.

\section{Declarations}

Funding No funding was received for this study.
Conflict of interest The authors declare no conflict of interest.

Availability of data and material The datasets generated during and/or analyzed during the current study are available from the corresponding author on reasonable request.

Code availability Not applicable.

Author contributions $\mathrm{IH}$ and $\mathrm{MN}$ are the guarantors of the paper; $\mathrm{IH}$, KGT, DJH, and MN contributed to the study design; $\mathrm{IH}, \mathrm{HH}, \mathrm{KS}$, and TS acquired the data; IH, KGT, DJH, and MN developed the analysis plan; KGT, and MN analyzed the data; IH, KGT, DJH, and MN interpreted the data; IH, KGT, and MN drafted the manuscript.

Ethics approval This study was approved by the Human Subjects Review Committee of National Hospital Organization Ibarakihigashi National Hospital (IRB number: 2017-027).

Consent to participate Not applicable.

Consent for publication Not applicable.

\section{References}

1. World Health Organization. Ageing: Global population. 2010 October 2, 2010. https://www.who.int/features/qa/72/en/. Accessed 22 Nov 2020 
2. Byng-Maddick R, Noursadeghi M. Does tuberculosis threaten our ageing populations? BMC Infect Dis. 2016;11(16):119.

3. Schaaf HS, Collins A, Bekker A, Davies PD. Tuberculosis at extremes of age. Respirology. 2010;15(5):747-63.

4. International Institute for Applied Systems Analysis (IIASA). Aging Demographic Data Sheet 2018. 2018. https://iiasa.ac.at/ web/home/research/researchPrograms/WorldPopulation/Publi cationsMediaCoverage/ModelsData/AgingDemDataSheet2018_ web.pdf. Accessed 22 Nov 2020.

5. Cabinet Office Japan. Annual Report on the Ageing Society [Summary] FY 2019. Japan; 2019.

6. Japan Anti-Tuberculosis Association. Statistics of TB in Japan 2018. Tokyo: JATA; 2018.

7. Japanese Society for Tuberculosis. Guide for tuberculosis (in Japanese). Tokyo: Nankodo; 2018.

8. Nahid P, Dorman SE, Alipanah N, Barry PM, Brozek JL, Cattamanchi A, et al. Official American Thoracic Society/Centers for Disease Control and Prevention/Infectious Diseases Society of America Clinical Practice Guidelines: treatment of drug-susceptible tuberculosis. Clin Infect Dis. 2016;63(7):e147-95.

9. Rousset S, Lafaurie M, Guet-Revillet H, Protin C, Le Grusse J, Derumeaux H, et al. Safety of pyrazinamide for the treatment of tuberculosis in older patients over 75 years of age: a retrospective monocentric cohort study. Drugs Aging. 2021;38(1):43-52.

10. Di Gennaro F, Vittozzi P, Gualano G, Musso M, Mosti S, Mencarini $P$, et al. Active pulmonary tuberculosis in elderly patients: a 2016-2019 retrospective analysis from an italian referral hospital. Antibiotics (Basel). 2020;9(8):489.

11. Gardner Toren K, Spitters C, Pecha M, Bhattarai S, Horne DJ, Narita M. Tuberculosis in older adults: Seattle \& King County, Washington. Clin Infect Dis. 2019;70(6):1202-7.

12. Hase I, Gardner Toren K, Hirano H, Yabuuchi Y, Kawashima $\mathrm{K}$, Kubota S, et al. Tuberculosis in older adults: Ibaraki, Japan [abstract]. Am J Respir Crit Care Med. 2020;201:A6373.

13. Centers for Disease Control and Prevention DoTE. CDC Tuberculosis Surveillance Data Training-Report of Verified Case of Tuberculosis (RVCT), Instruction Manual. 2009 September 1, 2012. https://www.cdc.gov/tb/programs/rvct/default.htm. Accessed 22 Nov 2020.

14. Lepakhin VK. Safety of medicines: a guide to detecting and reporting adverse drug reactions: why health professionals need to take action. Geneva: World Health Organization; 2002.
15. Gardner Toren K, Spitters C, Pecha M, Bhattarai S, Horne DJ, Narita M. Tuberculosis in older adults: Seattle and King County, Washington. Clin Infect Dis. 2020;70(6):1202-7.

16. Waitt CJ, Squire SB. A systematic review of risk factors for death in adults during and after tuberculosis treatment. Int $\mathrm{J}$ Tuberc Lung Dis. 2011;15(7):871-85.

17. Stead WW. The pathogenesis of pulmonary tuberculosis among older persons. Am Rev Respir Dis. 1965;91:811-22.

18. Mori T, Leung CC. Tuberculosis in the global aging population. Infect Dis Clin N Am. 2010;24(3):751-68.

19. Seto J, Wada T, Suzuki Y, Ikeda T, Mizuta K, Yamamoto T, et al. Mycobacterium tuberculosis transmission among elderly persons, Yamagata Prefecture, Japan, 2009-2015. Emerg Infect Dis. 2017;23(3):448-55.

20. Di Gennaro F, Gualano G, Timelli L, Vittozzi P, Di Bari V, Libertone $\mathrm{R}$, et al. Increase in tuberculosis diagnostic delay during first wave of the COVID-19 pandemic: data from an Italian Infectious Disease Referral Hospital. Antibiotics (Basel). 2021;10(3):272.

21. Narita M, Hatt G, Toren KG, Vuong K, Pecha M, Jereb JA, et al. Delayed tuberculosis diagnoses during the COVID-19 pandemic in 2020 - King County, Washington. Clin Infect Dis. 2021;ciab387. https://doi.org/10.1093/cid/ciab387.

22. Menzies D, Adjobimey M, Ruslami R, Trajman A, Sow O, Kim $\mathrm{H}$, et al. Four months of rifampin or nine months of isoniazid for latent tuberculosis in adults. N Engl J Med. 2018;379(5):440-53.

23. Sterling TR, Njie G, Zenner D, Cohn DL, Reves R, Ahmed A, et al. Guidelines for the treatment of latent tuberculosis infection: recommendations from the national tuberculosis controllers association and CDC, 2020. MMWR Recomm Rep. 2020;69(1):1-11.

24. Campbell JR, Trajman A, Cook VJ, Johnston JC, Adjobimey M, Ruslami R, et al. Adverse events in adults with latent tuberculosis infection receiving daily rifampicin or isoniazid: post-hoc safety analysis of two randomised controlled trials. Lancet Infect Dis. 2020;20(3):318-29. 